\title{
Dectin-1 stimulates IL-33 expression in dendritic cells via upregulation of IRF4
}

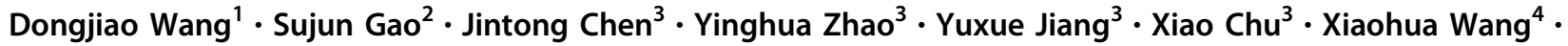 \\ Ning Liu ${ }^{1} \cdot$ Tianxue $\mathrm{Qin}^{2} \cdot$ Qing $\mathrm{Yi}^{3,5} \cdot$ Ying Yue ${ }^{1} \cdot$ Siqing Wang ${ }^{3}$
}

Received: 18 September 2017 / Revised: 11 January 2018 / Accepted: 24 January 2018 / Published online: 14 March 2018

(c) United States \& Canadian Academy of Pathology 2018

\begin{abstract}
Interleukin-33 (IL-33) is a potent contributor to antiviral immune responses and antitumor immunity. We recently discovered that IL-33 is overexpressed in dectin-1-activated dendritic cells (DCs). However, mechanisms of dectin-1-induced IL-33 expression in DCs remain elusive. Curdlan, an agonist of dectin-1, was used to mature DCs in this study. We found that dectin-1-induced IL-33 expression in DCs relies on Syk and Raf-1 pathways. By using nuclear factor (NF)- $\mathrm{KB}$ inhibitors, we

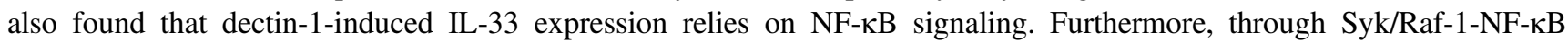
pathway, dectin-1 signaling stimulates DCs to overexpress interferon regulatory factor 4 (IRF4), which directly upregulates the expression of IL-33 in dectin-1-activated DCs. Thus, our study provides new insights into the mechanisms of dectin-1induced IL-33 expression in DCs and may provide new targets for improving DC-based cancer immunotherapy.
\end{abstract}

\section{Introduction}

Dendritic cells (DCs) are professional antigen-presenting cells and play a crucial role in antitumor immunity [1,2]. DCs exert the antitumor effects via the induction of tumorspecific effector $\mathrm{T}$ cells. Antitumor effector cells include $\mathrm{CD}^{+}$cytotoxic $\mathrm{T}$ lymphocytes (CTLs) and $\mathrm{CD} 4^{+} \mathrm{T}$ helper (Th) cells. CTLs are potent antitumor effector cells, mediating direct lysis of their target tumor cells. Antitumor Th cells include Th1, Th9 and Th17 cells. Among these, Th9

Ying Yue

yying119@126.com

$\triangle$ Siqing Wang siw1970@yahoo.com

1 Department of Gynecological Oncology, The First Hospital of Jilin University, Changchun 130061, China

2 Department of Hematology, The First Hospital of Jilin University, Changchun 130061, China

3 Department of Cancer Immunology, The First Hospital of Jilin University, Changchun 130061, China

4 Department of Internal Medicine, Linhai First People's Hospital, Linhai, Zhejiang 317000, China

5 Department of Cancer Biology, Lerner Research Institute, Cleveland Clinic, Cleveland, Ohio 44195, USA cells are shown to have better antitumor efficacy than Th1 and Th17 cells [3-5]. Cytokine milieu and DC costimulatory molecules are crucial for DCs to induce antitumor effector $\mathrm{T}$ cells. Therefore, strategies to stimulate $\mathrm{DC}$ expression of cytokines that favor the induction of antitumor effector cells may greatly increase the therapeutic efficacy of DC-based cancer immunotherapy.

Interleukin-33 (IL-33) is a cytokine of the IL-1 family and the ligand for ST2 receptor [6, 7]. IL-33 is a pleiotropic cytokine that regulates the activity of both innate and adaptive immune responses. IL-33 contributes substantially in multiple inflammatory diseases, such as asthma and atopic dermatitis, by stimulating the production of type II cytokines [8-10]. IL-33 drives protective antiviral CTL responses [11]. IL-33 also acts as an immunoadjuvant and augments antigenspecific antitumor immunity [12-14]. We recently found that dectin-1-activated DCs trigger potent Th9 cell responses and antitumor immunity $[15,16]$. In the mechanistic study, we identify 42 upregulated cytokines and costimulatory molecules in dectin-1-activated DCs, including IL-33 [16]. We also found that IL-33 contributes to dectin-1-activated DCinduced antitumor efficacy and Th9/1 cell differentiation (unpublished data). However, mechanisms of dectin-1induced IL-33 expression in DCs still remain unclear.

Dectin-1 activates Syk, Raf-1 and nuclear factor (NF)- $\mathrm{kB}$ signaling pathways [15-17]. In this study, we found that dectin-1 enhances interferon regulatory factor 4 (IRF4) 
expression through Syk, Raf-1 and NF-אB pathways, which consequently increases IL-33 expression in DCs. Our study provides new insights into the mechanisms of dectin-1induced IL-33 expression in DCs and may present new targets for improving DC-based cancer immunotherapy.

\section{Materials and methods}

\section{Mice and reagents}

C57BL/6 and Balb/c mice were purchased from the Jackson Laboratory. Dectin- $1^{-1-}$ mice were provided by G. Brown (University of Aberdeen, Aberdeen, Scotland). Mice were bred and maintained in pathogen-free facilities at the First Hospital Animal Center of Jilin University. 6 to 8-week-old mice were used for the experiments. All animal experimental procedures were reviewed and approved by the Animal Ethical Committee of First Hospital of Jilin University.

RPMI-1640 medium, penicillin and streptomycin were purchased from Invitrogen. Fetal bovine serum was purchased from Hyclone. Recombinant murine granulocytemacrophage colony-stimulating factor (GM-CSF), IL-4, IL$1 \beta$ and tumor necrosis factor-alpha (TNF- $\alpha$ ) were purchased from Peprotech. Recombinant human transforming growth factor-beta (TGF- $\beta$ ) was purchased from R\&D Systems. Functional anti-mouse CD3e and CD28 antibodies (mAbs) were purchased from eBioscience. Curdlan was purchased from Sigma-Aldrich. Lipopolysaccharide (LPS) and poly I:C were purchased from Sigma-Aldrich and Invivogen respectively. Syk inhibitor Piceatannol and Raf-1 kinase inhibitor GW5074 were purchased from Calbiochem. NF-kB inhibitor Bortezomib was purchased from Selleckchem. NF-кB inhibitor (CAS 213546-53-3) was purchased from Santa Cruz, and JSH-23 (a NF-KB inhibitor) was purchased from MCE.

\section{DC generation}

Mouse immature DCs (iDCs) were generated as described previously $[16,18]$. At day 7 , iDCs were collected and matured in RPMI-1640 complete medium containing TNF- $\alpha$ $\left(10 \mathrm{ng} \mathrm{mL}^{-1}\right)$ and IL-1 $\beta\left(10 \mathrm{ng} \mathrm{mL}^{-1}\right)$ (bone marrow-derived DCs, BMDCs) or Curdlan $\left(5 \mu \mathrm{g} \mathrm{m}^{-1}\right)$ (curdlan-activated iDCs, CurDCs). At day 9, mature DCs and culture supernatants were harvested for further analysis.

In some experiments, iDCs were treated with GM-CSF (10 $\left.\mathrm{ng} \mathrm{mL}^{-1}\right)$, poly I:C $\left(25 \mu \mathrm{g} \mathrm{m}^{-1}\right)$ or LPS $\left(10 \mathrm{ng} \mathrm{mL}^{-1}\right)$ for 2 days. Cells were harvested for IL-33 expression analysis.

In some experiments, iDCs were generated from wild-type (WT) and Dectin- $1^{-1-}$ C57BL/6 mice and matured with TNF$\alpha / \mathrm{IL}-1 \beta$ or Curdlan for 2 days. In some experiments, Piceatannol $(40 \mu \mathrm{M})$, GW5074 $(1 \mu \mathrm{M})$, Bortezomib (10 nM), NF$\kappa \mathrm{B}$ Inhibitor $\left(2 \mu \mathrm{g} \mathrm{mL}^{-1}\right)$ or JSH-23(30 $\left.\mu \mathrm{M}\right)$ was added during
DC maturation, Dimethyl Sulfoxide (DMSO) (0.1\%; Sigma) served as control. Cells were matured for 2 days. Mature DCs and culture supernatants were harvested for further analysis.

\section{qPCR and western blot analyses}

Quantitative PCR (qPCR) was performed as previously described [16]. Total RNA was extracted from cells by using an RNeasy Mini kit (Qiagen) according to the manufacturer's instructions. The mRNA levels of Il33, Irfl, Irf4, Irf7 and Il9 by $\mathrm{DCs}$ and $\mathrm{CD} 4^{+} \mathrm{T}$ cells were analyzed. Expression was normalized to the house-keeping gene Gapdh. Primer sets used for these analyses are: IL-33: forward 5'-TTCCAACTCCAAGATTTCCC- $3^{\prime}$ and reverse $5^{\prime}$-TGTCAACAGACGCAGCAAA-3'; Irf1: forward 5'-GCCGAGACACTAAGAGCAAA- $3^{\prime}$ and reverse 5'-TGTGGTCATCAGGTAGGGTAGA-3'; Irf4: forward 5'-AGCCCAGCAGGTTCATAACT- $3^{\prime}$ and reverse $5^{\prime}$-CTGGCAACCATTTTCACAAG3'; Irf7: forward 5'-CACAGCACAGGGCGTTTTA-3' and reverse 5'-AGACAAGCACAAGCCGAGAC-3'; Il9: forward 5'-GTGTCTCTCCGTCCCAACTGATG-3' and reverse 5'GATTTCTGTGTGGCATTGGTCAG-3'; and Gapdh: forward 5'-TGCACCACCAA CTGCTTAGC-3' and reverse 5'GGATGCAGG GATGATGTTCT-3'.

Western blot assay was performed as previously described [16]. Anti-mouse IRF4, $\beta$-actin antibodies and RIPA Buffer were purchased from Cell Signaling Technology (CST). Anti-mouse IRF7 was purchased from Abcam.

\section{Enzyme-linked immunosorbent assay (ELISA)}

ELISA was performed as previously described [19]. Capture and biotinylated antibodies against mouse IL-33 and recombinant mouse IL-33 standard were purchased from R\&D Systems. Avidin-HRP (horseradish peroxidase) was purchased from BioLegend.

\section{RNA interference}

RNA interference was performed as previously described [16]. In brief, at day 6 , iDCs were transfected with $50 \mathrm{nM}$ small interfering RNA (siRNA), silencing efficacy was confirmed by qPCR. At day 8, iDCs were matured for $48 \mathrm{~h}$, then mDCs were collected and analyzed by qPCR and ELISA. Syk, Rafl and control siRNAs were shown in the previous publication [16]. siRNAs used for Irf4 are: siRNA-1 sense: $5^{\prime}$ GCAAGUGUUUGCUCACCAU-3' and antisense 5'-AUGGUGAGCAAACACUUGC-3'; siRNA-2 sense: 5'-CCAGACAACUGUAUUACUU- $3^{\prime}$ and antisense $5^{\prime}$-AAGUAAUACAGUUGUCUGG- $3^{\prime}$; siRNA-3 sense: 5'-CCGUCAUUCUUCCAUCCAA- $3^{\prime}$ and antisense 5'-UUGGAUGGAAGAAUGACGG-3'. Il33 siRNAs (NM_133775) were purchased from Sigma-Aldrich. 


\section{Luciferase reporter assays}

Luciferase reporter assays were performed as previously described [16]. The luciferase reporter vector pGL4.10, a control vector pGL4.74 and expression vectors for NF- $\mathrm{KB}$ molecules p50, p65, c-Rel, p52 and RelB were purchased from Addgene. A 2112-bp mouse Il33 promoter was cloned from mouse genomic DNA using primers: forward primer, 5'-GCTCGCTAGCCTCGAGACAGAGTGAATGAGCAAAGAGG-3', and reverse primer, 5'-TCTTGATATCCTCGAGGATTCTGCCGTGATTTCTCC- $3^{\prime}$. The 2112 bp mouse Il33 promoter was inserted into pGL4.10 (mIl33pGL4.10). HEK293 $\mathrm{T}$ cells were transiently transfected with mIl33-pGL4.10 or pGL4.74 and expression vectors for $\mathrm{NF}-\mathrm{Kb}$ molecules by Lipofectamine 2000 (Invitrogen). Promoter activity was measured with Dual-Luciferase Reporter Assay System (Promega). Values are normalized to internal control and expressed as the mean \pm s.d. of relative luciferase units.

\section{Th9 cell differentiation}

CurDCs with or without Il33 and Irf4 siRNAs treatment were used to generate Th9 cells as previously described [16]. After 3 days of culture, cells were harvested for the analysis of $I l 9$ expression by qPCR.

\section{Statistical analysis}

The Student's $t$-test was used to compare various experimental groups. A $P$-value of $<0.05$ was considered significant.

\section{Results}

\section{Dectin-1 stimulates IL-33 expression via Syk and Raf-1}

IL-33 is overexpressed in dectin-1-activated DCs (Fig. 1a). Syk and Raf-1 are two independent downstream signaling
A

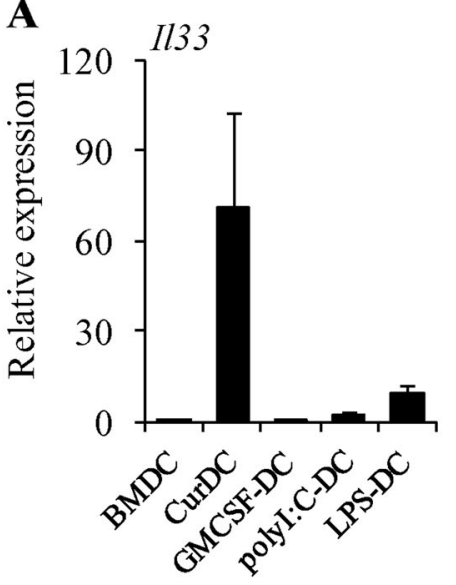

D

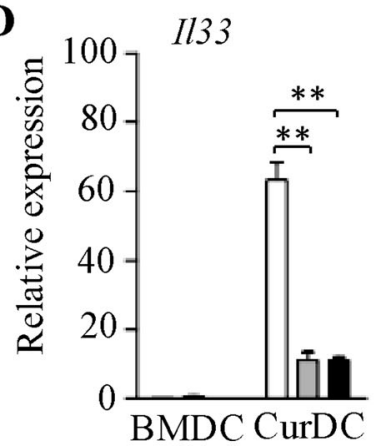

B

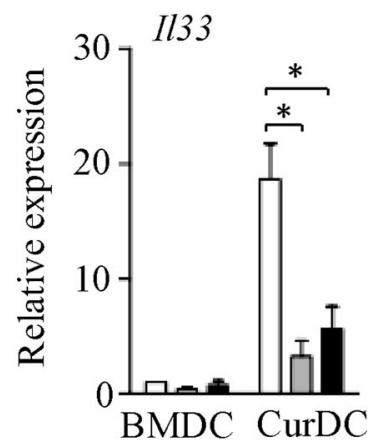

C

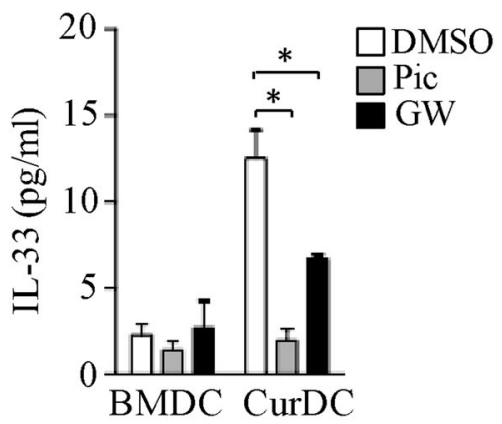

$\mathbf{E}$

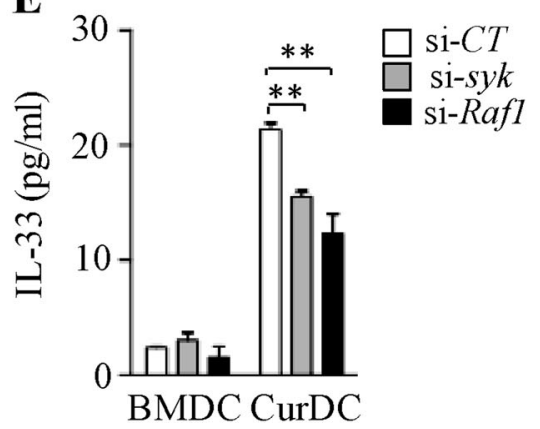

mRNA levels of $I I 33$ in DCs. $\mathbf{c}$ ELISA examined the protein levels of IL-33 in the culture supernatants. d, e Mouse iDCs were treated by Syk (si-Syk), Raf-1 (si-Rafl) or control siRNA (si-CT) and subjected to the stimulation of TNF $\alpha / I L-1 \beta$ (BMDCs) or Curdlan (CurDCs) for $48 \mathrm{~h}$. qPCR $\mathbf{d}$ and ELISA e assessed IL-33 expression by DCs. Results shown are the mean $\pm \mathrm{SD}$ of at least three independent experiments. $* P<0.05 ; * * P<0.01$ 
pathways induced by dectin-1 in DCs [17]. To investigate the role of Syk and Raf-1 signaling in dectin-1-induced IL33 expression, Syk-specific inhibitor Piceatannol and Raf-1specific inhibitor GW5074 were used during DC maturation. The inhibition of Syk or Raf-1 in DCs significantly decreased IL-33 expression induced by a dectin-1 agonist Curdlan (Figs. 1b, c). In addition, by using siRNAs to specifically silence Syk or Rafl in mouse DCs, we found that knockdown of either Syk or Rafl in DCs reduced IL-33 expression induced by Curdlan (Figs. 1d, e). These results showed that dectin-1-induced IL-33 expression through Syk and Raf-1 signaling pathways.

\section{NF-KB is indirectly involved in dectin-1-induced IL- 33 expression}

Dectin-1 activates NF-kB via Syk and/or Raf-1 pathways [17]. To investigate the role of NF- $\mathrm{kB}$ signaling in dectin-1induced IL-33 expression, Bortezomib was used during DC maturation. Bortezomib is a proteasome inhibitor, it was used to inhibit NF-kB [20]. We found that mouse DCs matured by Curdlan plus bortezomib expressed less IL-33 than cells matured by Curdlan alone (Figs. 2a, b). To confirm the results, other two NF- $\kappa B$ inhibitors, NF- $\kappa B$ Inhibitor (CAS 213546-53-3) and JSH-23, were used. As shown in Fig. 2c, inhibiting NF- $\mathrm{KB}$ by either NF- $\mathrm{KB}$ Inhibitor or JSH-23 largely abrogated IL-33 expression in CurDCs. These results indicate that dectin-1-induced IL-33 expression through NF- $\mathrm{KB}$ signaling pathway.

To further determine the role of NF- $\kappa B$ pathway in dectin-1-induced IL-33 expression, we performed luciferase reporter assays to examine whether these NF- $\mathrm{\kappa B}$ transcription factors could bind directly to Il33 promoters and activate their expression. As shown in Fig. 2d, only RelB was detected to slightly bind to and activate Il33 promoter. These results indicated that NF- $\mathrm{kB}$ is indirectly involved in dectin-1-induced IL-33 expression and suggested that some other factors that regulate IL-33 expression might be induced through dectin-1-triggered Syk, Raf-1 and NF-kB signaling pathways.
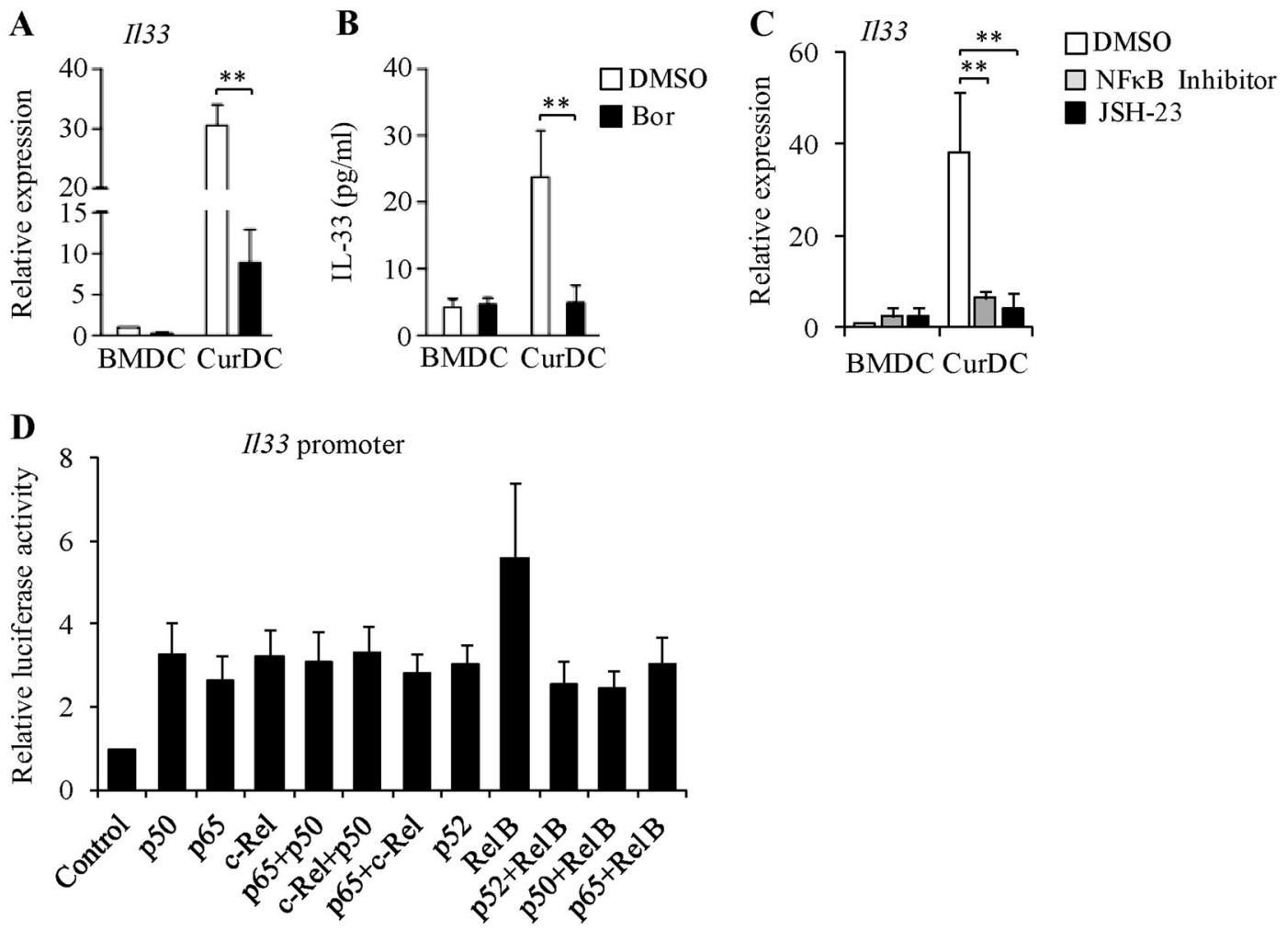

Fig. 2 Active NF- $\kappa B$ is indirectly involved in dectin-1-induced IL-33 expression. a, b Mouse iDCs were matured by TNF $\alpha /$ IL-1 $\beta$ (BMDC) or Curdlan (CurDC) in the absence (DMSO) or presence of NF- $\kappa \mathrm{B}$ inhibitor bortizomib (Bor) for $48 \mathrm{~h}$. qPCR a and ELISA $\mathbf{b}$ assessed IL33 expression by DCs. c Mouse iDCs were matured by TNF $\alpha / \mathrm{IL}-1 \beta$ (BMDC) or Curdlan (CurDC) in the absence (DMSO) or presence of NFKB Inhibitor or JSH-23 for $48 \mathrm{~h}$. qPCR assessed IL-33 expression by DCs. d 293 T cells were transiently transfected with vectors contained Il33 promoter or empty vector as a control, followed by transfecting with vectors expressing the indicated NF- $\mathrm{\kappa B}$ molecules. Luciferase reporter assay showed NF- $\kappa$ B-dependent activation of IL33 promoter in $293 \mathrm{~T}$ cells. Data are presented as mean \pm SD of at least three independent experiments. $* * P<0.01$ 
A

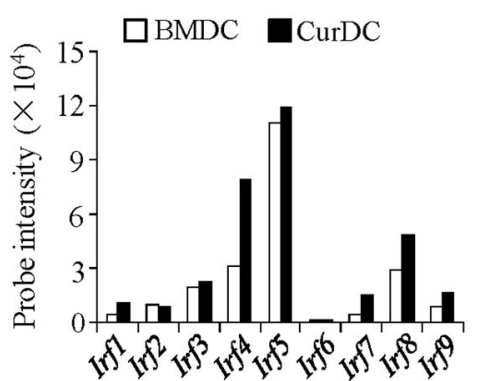

B

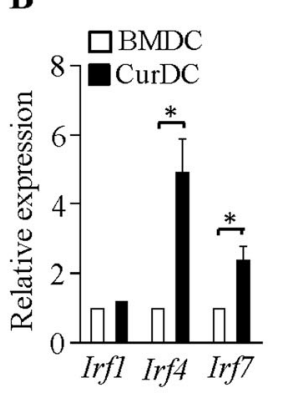

C

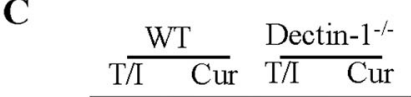

IRF4

IRF7

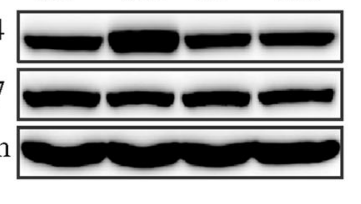

D $\quad$ Irf4

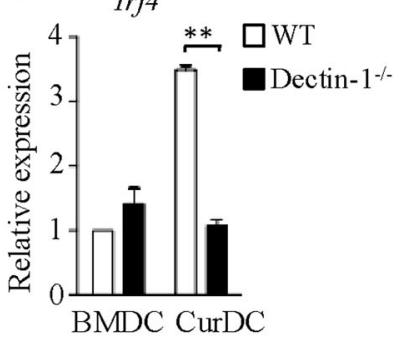

Fig. 3 Dectin-1 stimulates DCs to express IRF4. a The abundance of mRNA levels of IRF family members in BMDCs and CurDCs extracted from microarray gene expression data. b qPCR assessed mRNA levels of Irf1, Irf4 and Irf7 in BMDCs and CurDCs. c Western blots analyzed the protein levels of IRF4 and IRF7 in BMDCs (T/I) or CurDCs (Cur) generated from WT and dectin-1 ${ }^{-1-}$ mice. $\beta$-Actin was used as loading controls. d qPCR assessed mRNA levels of Irf4 in BMDCs or CurDCs generated from WT and dectin-1 ${ }^{-1-}$ mice. Data are presented as mean \pm SD of at least three $\mathbf{b}, \mathbf{d}$ independent experiments or representative of three $\mathbf{c}$ independent experiments. $* P<0.05 ; * * P<0.01$

A

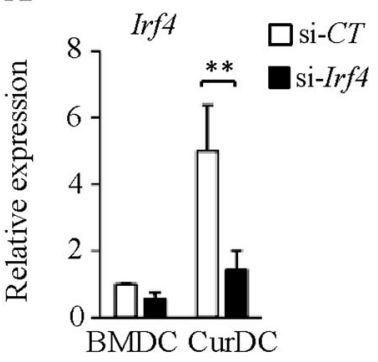

B

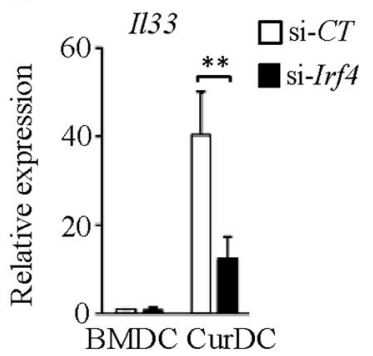

C

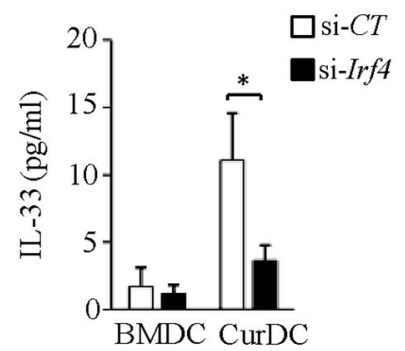

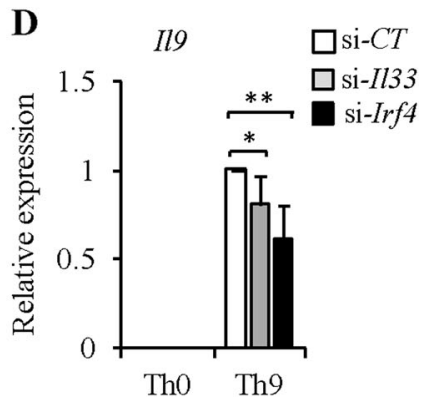

Fig. 4 Dectin-1-induced IL-33 relies on IRF4 expression. Mouse iDCs were treated by Irf4 siRNA (si-Irf4) or control siRNA (si-CT) and stimulated by TNF $\alpha / \mathrm{IL}-1 \beta$ (BMDCs) or Curdlan (CurDCs) for $48 \mathrm{~h}$. qPCR assessed the expression of Irf4 $\mathbf{a}$ and Il33 $\mathbf{b}$ in DCs. $\mathbf{c}$ ELISA examined the protein levels of IL-33 in the culture supernatants. d Mouse iDCs were treated by Il33 siRNA (si-Il33), Irf4 siRNA (si-Irf4)

\section{Dectin-1 stimulates DCs to express IRF4}

To investigate the potential transcription factors that are triggered by dectin- 1 and regulate IL-33 expression, we reanalyzed the microarray gene expression data of CurDCs and BMDCs (GSE81111) [16]. We observed increased expression of IRF family member genes in CurDCs compared with BMDCs, especially Irf4 and Irf7 (Fig. 3a). The upregulation of Irf4 mRNA, Irf7 mRNA and IRF4 protein in CurDCs compared with BMDCs was confirmed by qPCR and western blots (Figs. 3b, c), whereas the expression of IRF7 protein remained unchanged (Fig. 3c). Furthermore, the upregulation of IRF4 expression was completely abolished in CurDCs generated from dectin- $1^{-/-}$mice (Figs. 3c, d). These results showed that dectin-1 signaling enhanced IRF4 expression in DCs.

\section{Dectin-1 signaling increases IL-33 expression via IRF4}

We next investigated the role of IRF4 in dectin-1-induced expression of IL-33. Irf4-specific siRNAs were used to

or control siRNA (si-CT) and stimulated by Curdlan for $48 \mathrm{~h}$. DCs were cocultured with naïve $\mathrm{CD}^{+}{ }^{+} \mathrm{T}$ cells under $\mathrm{Th} 9$-polarizing conditions. Cell cultures without (Th0) addition of Th9-polarizing cytokines TGF- $\beta$ and IL-4 were used as controls. Cells were cultured for 3 days. qPCR analysis of $I l 9$ in T cells. Data are presented as mean \pm $\mathrm{SD}$ of three independent experiments. ${ }^{*} P<0.05$; $* * P<0.01$

silence Irf4 in mouse DCs (Fig. 4a). We found that knockdown of Irf4 in DCs inhibited IL-33 expression induced by Curdlan (Figs. 4b, c). These results showed that dectin-1-induced IL-33 expression through the upregulation of IRF4.

We previously discovered that dectin-1-activated DCs trigger potent Th9 cell response [16]. To examine the role of IRF4 and IL-33 in the induction of Th9 cells by dectin-1activated DCs, CurDCs treated by Irf4 (si-Irf4) or Il33 (siIl33) siRNAs were used for Th9 cell induction in vitro. Th9 cells induced by si-Irf4 or si-Il33-treated CurDCs expressed lower levels of $I l 9$ than those induced by control CurDCs (Fig. 4d), indicating that IRF4 and IL-33 contribute dectin1-activated DC-induced Th9 differentiation.

\section{Dectin-1 drives IRF4 expression via Syk, Raf-1 and NF-KB pathways}

To examine the role of Syk, Raf-1 and NF- $\mathrm{kB}$ in dectin-1induced IRF4 expression, Syk, Raf-1 and NF- NF-kB inhibitors were used during DC maturation. The inhibition of Syk, Raf-1 or NF-kB in DCs significantly decreased 
A

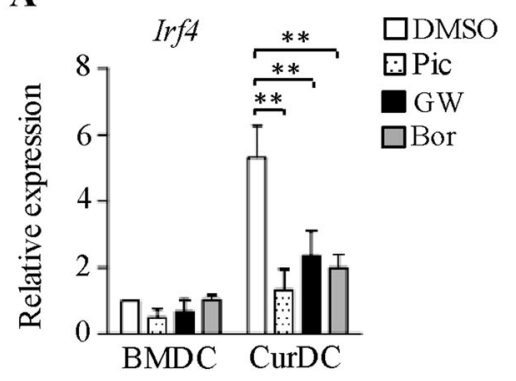

B

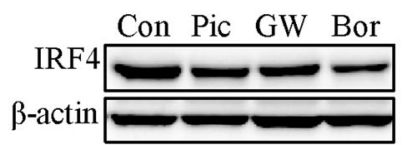

C

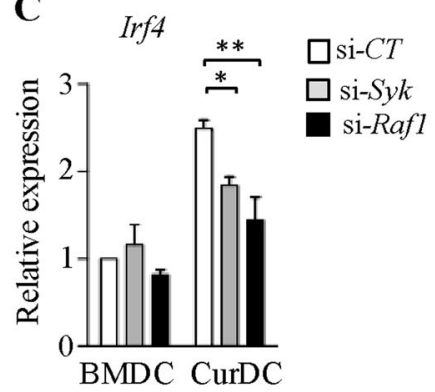

D

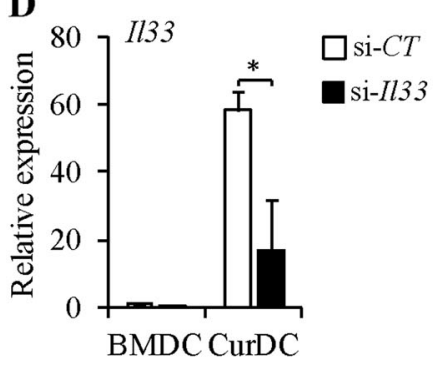

E

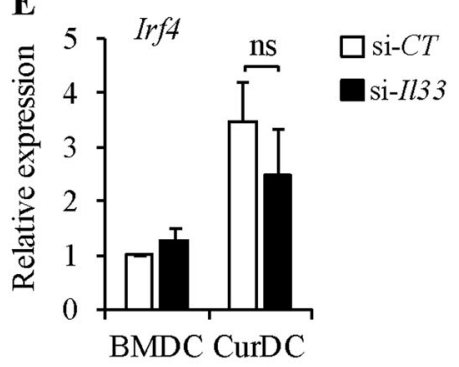



Fig. 5 Dectin-1 induces IRF4 via Syk/Raf-1 and NF-кB pathways. a, b Mouse iDCs were matured by TNF $\alpha / \mathrm{IL}-1 \beta$ (BMDCs) or Curdlan (CurDCs) in the presence of piceatannol (Pic), GW5074 (GW), bortezomib (Bor) or DMSO as controls for $48 \mathrm{~h}$. qPCR a and western blots $\mathbf{b}$ assessed IRF4 mRNA and protein expression in DCs. $\mathbf{c}$ Mouse iDCs were treated by Syk (si-Syk), Rafl (si-Rafl) or control siRNA (Si$C T$ ) and stimulated by TNF $\alpha / \mathrm{IL}-1 \beta$ (BMDCs) or Curdlan (CurDCs) for $48 \mathrm{~h}$. qPCR analyzed Irf4 expression in DCs. d, e Mouse iDCs

IRF4 expression induced by Curdlan (Figs. 5a, b). In addition, knockdown of either Syk or Rafl by their specific siRNAs in DCs reduced Irf4 expression induced by Curdlan (Fig. 5c). In contrast, knockdown of Il33 in DCs does not affect IRF4 expression in CurDCs (Figs. 5d, e). These results showed that dectin-1-induced IRF4 expression through Syk, Raf-1 and NF-kB signaling pathways.

\section{Discussion}

IL-33 is overexpressed in dectin-1-activated DCs and it plays an important role in dectin-1-activated DC-induced antitumor immunity $[15,16]$. Therefore, clarifying the mechanisms of dectin-1-induced IL-33 expression may have important clinical significance. In this study, we found that dectin-1 activates Syk, Raf-1 and NF-kB downstream signaling pathways, which consequently result in increased expression of IL-33 in DCs. This result was confirmed by using Syk, Raf-1 and NF- $\mathrm{kB}$ inhibitors and/or siRNAs for silencing $S y k$ or Raf- 1 during DC maturation as these treatments inhibit IL-33 expression in dectin-1-activated DCs. These results are consistent with previous were treated by $I l 33$ (si-Il33) or control siRNA (Si-CT) and stimulated by TNF $\alpha / \mathrm{IL}-1 \beta$ (BMDCs) or Curdlan (CurDCs) for $48 \mathrm{~h}$. qPCR analyzed Il33 d and Irf4 e expression in DCs. f Schema showing the signaling pathways of dectin-1-induced IL-33 expression in DCs. Data are presented as mean $\pm \mathrm{SD}$ of at least three $\mathbf{a}, \mathbf{c}-\mathbf{e}$ independent experiments or representative of three $\mathbf{b}$ independent experiments. $* P$ $<0.05 ; * * P<0.01$

observations that $\beta$-glucan recognition via dectin-1 led to the induction of IL-33 [21] and NF- $\mathrm{KB}$ signaling is involved in IL-33 expression induced by Toll-like receptor 5 in DCs [22]. Thus, our data demonstrated Syk, Raf- 1 and NF- $\mathrm{kB}$ as the downstream signaling pathways of dectin-1 in increasing IL-33 expression in DCs.

In this study, we found that none of NF- $\mathrm{KB}$ transcription factors could bind and activate the promoter of $I l 33$ gene, suggesting that dectin-1 downstream NF- $\mathrm{KB}$ signaling indirectly regulates IL-33 expression. IRF transcription factors IRF3, IRF4 and IRF7 are shown to induce IL-33 expression [23-25]. We found that dectin-1 enhanced the expression of IRF1, IRF4 and IRF7, especially IRF4, suggesting that dectin-1 induces IL-33 expression via upregulation of IRF4. Indeed, knockdown of IRF4 largely abolished dectin-1-induced IL-33 expression in DCs. Furthermore, dectin-1 agonist-induced IRF4 expression relies on dectin-1 and its downstream Syk, Raf-1 and NF-kB pathways. Thus, our data identified IRF4 as the major transcription factor for dectin-1-induced IL-33 expression.

In summary, our study demonstrates that dectin- 1 signaling in DCs activated Syk, Raf-1 and NF- $\mathrm{kB}$ pathways and enhanced the expression of IRF4, which resulted in 
increased expression of IL-33 (Fig. 5f). Our study provides new insights into the mechanisms of dectin-1-induced IL-33 expression in DCs and may present new targets for improving DC-based cancer immunotherapy.

Acknowledgements This work was supported by grants from National Natural Science Foundation of China (no. 81372536 for SW, 81502452 for XC and 81602485 for YZ) and a grant from the Natural Science Foundation of Jilin Province (no. 20170520006JH for XC).

\section{Compliance with ethical standards}

Conflict of interest The authors declare that they have no conflict of interest.

\section{References}

1. Garg AD, Coulie PG, Van den Eynde BJ, et al. Integrating nextgeneration dendritic cell vaccines into the current cancer immunotherapy landscape. Trends Immunol. 2017;38:577-93.

2. Sabado RL, Bhardwaj N. Cancer immunotherapy: dendritic-cell vaccines on the move. Nature. 2015;519:300-1.

3. Lu Y, Hong B, Li H, et al. Tumor-specific IL-9-producing CD8 + Tc9 cells are superior effector than type-I cytotoxic Tc1 cells for adoptive immunotherapy of cancers. Proc Natl Acad Sci USA. 2014;111:2265-70.

4. Lu Y, Hong $\mathrm{S}, \mathrm{Li} \mathrm{H}$, et al. Th9 cells promote antitumor immune responses in vivo. J Clin Invest. 2012;122:4160-71.

5. Purwar R, Schlapbach C, Xiao S, et al. Robust tumor immunity to melanoma mediated by interleukin-9-producing T cells. Nat Med. 2012;18:1248-53.

6. Molofsky AB, Savage AK, Locksley RM. Interleukin-33 in tissue homeostasis, injury, and inflammation. Immunity. 2015;42:1005-19.

7. Liew FY, Pitman NI, McInnes IB. Disease-associated functions of IL-33: the new kid in the IL-1 family. Nat Rev Immunol. 2010;10:103-10.

8. de Kleer IM, Kool M, de Bruijn MJ, et al. Perinatal activation of the interleukin-33 pathway promotes type 2. Immun Dev Lung Immun. 2016;45:1285-98.

9. Cayrol C, Girard JP. IL-33: an alarmin cytokine with crucial roles in innate immunity, inflammation and allergy. Curr Opin Immunol. 2014;31:31-37.

10. Cohen ES, Scott IC, Majithiya JB, et al. Oxidation of the alarmin IL-33 regulates ST2-dependent inflammation. Nat Commun. 2015;6:8327.
11. Bonilla WV, Frohlich A, Senn K, et al. The alarmin interleukin-33 drives protective antiviral $\mathrm{CD} 8(+) \mathrm{T}$ cell responses. Science. 2012;335:984-9.

12. Villarreal DO, Wise MC, Walters JN, et al. Alarmin IL-33 acts as an immunoadjuvant to enhance antigen-specific tumor immunity. Cancer Res. 2014;74:1789-1800.

13. Mayuzumi N, Matsushima H, Takashima A. IL-33 promotes DC development in BM culture by triggering GM-CSF production. Eur J Immunol. 2009;39:3331-42.

14. Rank MA, Kobayashi T, Kozaki H, et al. IL-33-activated dendritic cells induce an atypical TH2-type response. J Allergy Clin Immunol. 2009;123:1047-54.

15. Chen J, Zhao Y, Chu X, et al. Dectin-1-activated dendritic cells: a potent $\mathrm{Th} 9$ cell inducer for tumor immunotherapy. Oncoimmunology. 2016;5:e1238558.

16. Zhao Y, Chu X, Chen J, et al. Dectin-1-activated dendritic cells trigger potent antitumour immunity through the induction of Th9 cells. Nat Commun. 2016;7:12368.

17. Gringhuis SI, den Dunnen J, Litjens M, et al. Dectin-1 directs T helper cell differentiation by controlling noncanonical NF-kappaB activation through Raf-1 and Syk. Nat Immunol. 2009;10:203-13.

18. Joo H, Upchurch $\mathrm{K}$, Zhang $\mathrm{W}$, et al. Opposing roles of dectin-1 expressed on human plasmacytoid dendritic cells and myeloid dendritic cells in Th2 polarization. J Immunol. 2015; 195:1723-31.

19. Chen L, Wang S, Zhou Y, et al. Identification of early growth response protein 1 (EGR-1) as a novel target for JUN-induced apoptosis in multiple myeloma. Blood. 2010;115:61-70.

20. Vegran F, Berger H, Boidot R, et al. The transcription factor IRF1 dictates the IL-21-dependent anticancer functions of TH9 cells. Nat Immunol. 2014;15:758-66.

21. Lilly LM, Gessner MA, Dunaway CW, et al. The beta-glucan receptor dectin-1 promotes lung immunopathology during fungal allergy via IL-22. J Immunol. 2012;189:3653-60.

22. Su Z, Lin J, Lu F, et al. Potential autocrine regulation of interleukin-33/ST2 signaling of dendritic cells in allergic inflammation. Mucosal Immunol. 2013;6:921-30.

23. Polumuri SK, Jayakar GG, Shirey KA, et al. Transcriptional regulation of murine IL-33 by TLR and non-TLR agonists. J Immunol. 2012;189:50-60.

24. Williams JW, Tjota MY, Clay BS, et al. Transcription factor IRF4 drives dendritic cells to promote Th2 differentiation. Nat Commun. 2013;4:2990.

25. Sun L, Zhu Z, Cheng N, et al. Serum amyloid A induces interleukin-33 expression through an IRF7-dependent pathway. Eur J Immunol. 2014;44:2153-64. 